九州大学学術情報リポジトリ

Kyushu University Institutional Repository

\title{
Simulation of a UV-C Conveyor System Using Computational Fluid Dynamics Techniques on the Uniformity of the Incident UV-C Dose Distribution to Strawberry
}

\section{KINGWASCHARAPONG, Passakorn}

Laboratory of Postharvest Science, Division of Bioproduction Environmental Sciences, Department of Agro-environmetal Sciences,

\section{IIDA, Yuki}

Laboratory of Postharvest Science, Division of Bioproduction Environmental Sciences, Department of Agro-environmetal Sciences,

TANAKA, Fumina

Graduate school of Bioresource and Bioenvironmental Sciences, Kyushu University

TANAKA, Fumihiko

Graduate school of Bioresource and Bioenvironmental Sciences, Kyushu University

https://doi.org/10.5109/4103903

出版情報: 九州大学大学院農学研究院紀要. 65 (2)，pp.371-378，2020-09. Faculty of Agriculture， Kyushu University

バージョン :

権利関係 : 


\title{
Simulation of a UV-C Conveyor System Using Computational Fluid Dynamics Techniques on the Uniformity of the Incident UV-C Dose Distribution to Strawberry
}

\author{
Passakorn KINGWASCHARAPONG ${ }^{1}$, Yuki IIDA ${ }^{1}$, Fumina TANAKA ${ }^{2}$ \\ and Fumihiko TANAKA ${ }^{2 *}$
}

\author{
Laboratory of Postharvest Science, Division of Bioproduction Environmental Sciences, \\ Department of Agro-environmental Sciences, Faculty of Agriculture, \\ Kyushu University, Fukuoka 819-0395, Japan \\ (Received May 22, 2020 and accepted May 27, 2020)
}

\begin{abstract}
This study aimed to evaluate and improve the uniformity of the UV-C dose distribution and microbial inactivation on the surface of strawberries using computational fluid dynamics (CFD) simulation technique. Eight different UV-C configuration models were designed. The pattern of UV-C lamps and their covers affected the intensity of UV-C, whereas adding reflectors into the model resulted in better uniformity of the UV-C dose. Model H (lamps and theirs covers placed at the corners of the chamber and 4 reflectors added between the lamps) provided the best uniformity of the incident UV-C dose. Longer treatment time (6 min) enhanced the inhibition of Penicillium digitatum. Therefore, simulation using CFD techniques may be a promising method to predicting UV-C irradiation parameters.
\end{abstract}

Keywords: Inactivation, Modelling, Penicillium digitatum, Ultraviolet- C configurations, Ultraviolet- C treatment

\section{INTRODUCTION}

Nowadays, many technologies are used in the food industry to provide more diverse industrial food products and more competitive and efficient processes (Barbosa Cánovas et al., 2011). Although all technologies are increasingly being used, and not only conventional technologies but also immerging technologies, non-uniformity within processes is still being encountered (Knoerzer et al., 2011, Lu et al., 2010). Non-uniformity in treatment processes is an important concern due to its direct relation with food quality (Tanaka et al., 2016). Food safety issues can occur when microorganisms (especially foodborne pathogens) are not completely inactivated, as well as quality issues (Pan et al., 2018). Pu and Sun (2017) pointed out that non-uniformity in hot air drying and microwave drying treatments could influence the color of mango fruit and shorten its shelf-life.

Among new physical technologies, one of the most popular methods used in the food industry is ultravioletC (UV-C) irradiation (wavelength 100-280 nm) (Gutiérrez et al., 2018). The benefits of using UV-C treatment in food processing lines center on it being cheap, non-toxic, ecofriendly, and easy incorporate with other methods (Trivittayasil et al., 2016). Nevertheless, UV-C still has some limitations because it is difficult to achieve a uniform irradiation dose over complicated surfaces.

Recently, computational fluid dynamics (CFD) has

\footnotetext{
Laboratory of Postharvest Science, Division of Bioproduction Environmental Sciences,

Department of Agro-environmetal Sciences,

${ }^{2}$ Graduate school of Bioresource and Bioenvironmental Sciences, Kyushu University

* Corresponding authors: Fumihiko Tanaka (E-mail: fumit@ bpes.kyushu-u.ac.jp)
}

been applied as a numerical method and algorithm in food processing for analyzing problems involving nonuniformity of fluid flows, irradiation, etc. (Tanaka et al., 2016, Park and Yoon, 2018, Ambaw et al., 2013, Zhao et al., 2016). CFD techniques have been applied in many industries including heating (drying, cooking, sterilization, chilling) (Cârlescu et al., 2017), mass transfer (transpiration or dissolution) (Hou et al., 2018), phase change (freezing, melting, or boiling) (Kumarasamy et al., 2016), chemical reactions (combustion or rusting), and mechanical movement (impellers, pistons, fans or rudders) (Park and Yoon, 2018). However, there have been few reports using CFD modeling for predicting radiation intensity in terms of post-harvest quality. Trivittayasil et al. (2016) showed that a 4-lamp model with a horizontal distance of $300 \mathrm{~mm}$ between the lamps gave the most uniform dose distribution, with a mean radiation intensity of $2.00 \mathrm{~W} / \mathrm{m}^{2}$. They also validated the model theoretically using a geometrical approach. Tanaka et al. (2016) developed a three-dimensional irradiation model based on a discrete ordinates (DO) method to evaluate UV-C dose distribution on the surface of strawberries. They found that four lamps installed parallel to the direction of movement provided a uniform dose distribution. However, since Tanaka et al. (2016) and Trivittayasil et al. (2016) carried out their simulation based on an ideal model and discussed how to improve the uniformity of UV-C dose distribution on the surface of strawberries, the application of their findings has been limited. In this work we developed an actual simulation model (size, shape, position of lamp, etc.) based on a real shape and performance of UV-C treatment, because it is necessary to conduct research based on an actual model using CFD techniques in order to generate information that can be applied in the food industry. Furthermore, CFD application can reduce the research time for users as well as experimentation costs. 
The objectives of this study were to: 1) create a UV-C treatment model including a conveyor system based on an actual machine (size, shape, and operation setting) using CFD techniques, 2) design the optimal conditions using simulation techniques for surface decontamination of strawberry.

\section{MATERIAL AND METHODS}

The CFD simulation process consisted of 4 main steps including creating geometry, mesh, set up boundary conditions, and obtaining post-calculation results (Trivittayasil et al., 2015). In this study we developed eight different model configurations to improve the uniformity associated with UV-C irradiation, as described below.

\section{Geometry}

The geometry of a strawberry was obtained using a 3D laser scanner (Next Engine, USA). Generally, the different configuration models of UV-C treatment systems lead to difference in the uniformity of UV-C intensity inside the treatment chamber. The advantage of uniform UV-C irradiation throughout the process is gained through uniform product quality.

All models (A, B, C, D, E, F, G, and H) were created based on the actual geometry of a UV-C treatment system by using ANSYS Workbench 18.1 (ANSYS Inc., USA). The chamber sizes of all models (A, B, C, D, E, F, $\mathrm{G}$, and $\mathrm{H}$ ) were designed with width $400 \mathrm{~mm}$, height $500 \mathrm{~mm}$, and depth $1000 \mathrm{~mm}$ (Fig. 1A).

For Models A, B, and C, these models consisted of 8 UV-C lamps obtained from Panasonic (GL 30, specification $13.4 \mathrm{~W}$, length $893 \mathrm{~mm}$ ), which were placed in 4 pairs (2 lamps per pair). Each pair of lamps was covered with a lamp cover. Three pairs of lamps with a cover were placed at the top of the conveyor chamber at a distance of $97 \mathrm{~mm}$ from the sample (9 strawberries placed in a tray), and one pairs of lamps and cover were placed at the bottom of the chamber. The distance between the bottom lamps in Models A, B, and C and the sample were 50, 30, and $10 \mathrm{~mm}$, respectively (Fig. 2A, $2 \mathrm{~B}$ and $2 \mathrm{C}$ ). The simulated nine strawberries placed in a low-density polyethylene (LLDPE) tray (B9, Yurikago, Oshiri Sangyo Co. Ltd.) used as the sample. The UV-C transmittance of LLDPE is 0.76. This sample was considered as being on a conveyor, with further details in the simulation section.

For Models D and E, these were adapted from Model A and included adding 2 reflectors $(65 \mathrm{~mm} \times 873 \mathrm{~mm})$ to these models. For Model D, the reflectors were placed at the top (left and right side) of the conveyor chamber

A

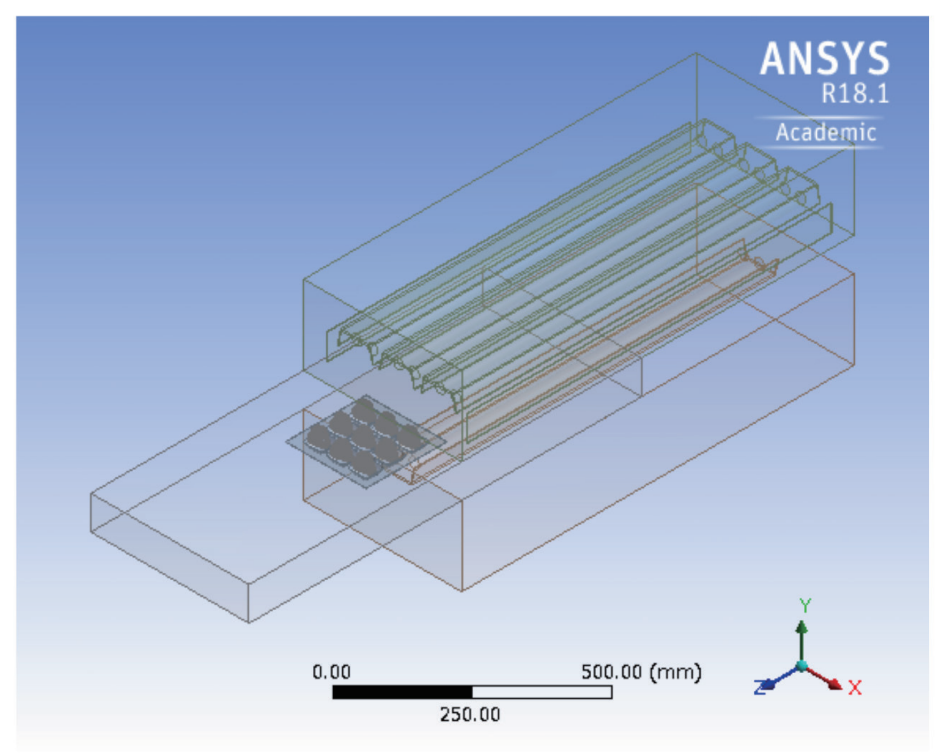

B
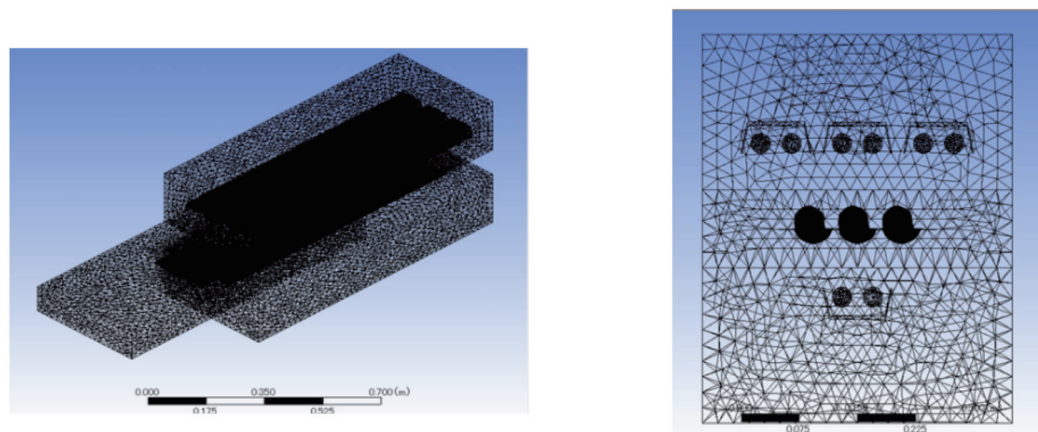

Fig. 1. The selected 3D model (A) meshing of 3D (B) model of UV-C conveyor configuration. 
at a distance of $47 \mathrm{~mm}$ from the sample (Fig. 2D). For Model E, the reflectors were placed at the bottom (left and right side) of the conveyor chamber also at $47 \mathrm{~mm}$ from the sample (Fig. 2E).

For Models $\mathrm{F}$ and $\mathrm{G}$, the position of the 4 pairs of lamps and their covers were rearranged. In Model F, 2 lamps and their covers were placed at the top, bottom, left, and right sides of the conveyor chamber, with the distances between the lamps (top, bottom, left and right) and the sample set as 97, 50, 50, and $50 \mathrm{~mm}$, respectively (Fig. 2F). In Model G, 2 lamps and their covers were each placed at the corners of the conveyor chamber, in which the radius of the lamp and distances from the sample are shown in Fig. 2G.

For Model H, this model adapted Model G by adding
4 reflectors placed between the lamps, as shown in Fig. $2 \mathrm{H}$.

\section{Meshing}

The geometries were then imported into ANSYS Meshing, which is a component of the ANSYS Workbench software (ANSYS Inc., USA) to create a volume mesh. The default automatic meshing method (Fig. 1B) was employed in accordance with the results of our previous study (Trivittayasil et al., 2016). Creating the most appropriate mesh can be used to achieve good simulation results. The resulting numbers of mesh elements were 2782 472, 2748 038, 2728 720, 2953 637, 2936 568, 2776 842, 3015 620, and 3020345 for Models A, B, $\mathrm{C}, \mathrm{D}, \mathrm{E}, \mathrm{F}, \mathrm{G}$, and $\mathrm{H}$, respectively. In order to simulate
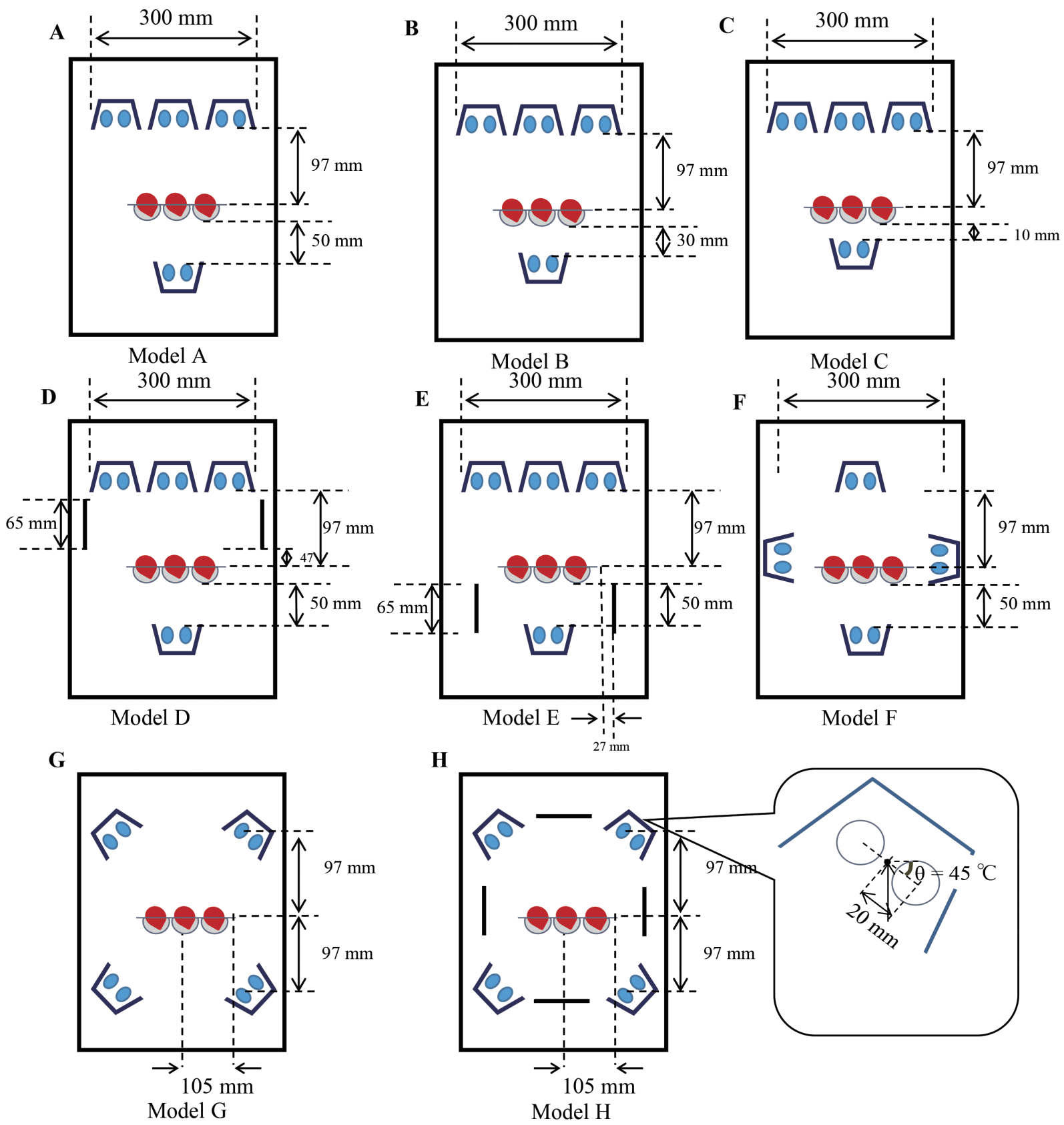

Fig. 2. UV-C conveyor configuration models. 
continuous UV-C treatment, the models were set to have a movement character using a sliding mesh technique, which was reported in accordance with the previous report Tanaka et al. (2016). Briefly, the model was divided to three cell zones, designated as: top, middle, and bottom, as shown in Fig. 1. The top and bottom zones of the models included the UV-C lamps with covers. The middle cell zone included the LLDPE tray with nine strawberries. These three cell zones of the model were used to simulate the movement characteristics of the conveyor. Mesh interface techniques were used for the boundary of each cell zone. Accordingly, the geometry of the middle zone was complicated, because it was fixed to be a stationary zone and the other zones (top and bottom) were created as moving zones. In the nonsteady simulation, all the computational domains, set as stationary, were set in one inertial reference frame. The motion of the boundary zones was described using profiles related to the time-dependent position of the moving objects.

\section{Set up boundary condition}

The simulation was carried out using Fluent 18.1 software (ANSYS Inc., USA), employing a DO radiation model with theta and phi divisions of $10 \times 10$ and theta and phi pixels of $3 \times 3$ to solve the radiative transfer equation in the computational domain. The actual intensity of UV-C lamp was $94.66 \mathrm{~W} / \mathrm{m}^{2}$ measured by a UV-C light meter (UVC-254, ASONE CO., Japan).

For completeness of the mathematical description, a set of boundary conditions was defined on the physical surfaces and domain walls, and important parameters are indicated in Table 1. To minimize the effect of thermal radiation from temperature differences between the sample and the lamps, which have power being applied to them, the temperature of the fluid was reduced to $10 \mathrm{~K}$ in accordance with Trivittayasil et al. (2016).

\section{Determination of optimum calculated step}

Model C was selected to assess the effect of the calculation step and UV-C intensity distribution on the sur- face of strawberries. The geometry, meshing, and set up boundary condition of Model $\mathrm{C}$ was created as described previously. The different calculation steps of Model $\mathrm{C}$ were set and named as Model C, Model C-1, and Model C-2 for $0.1,0.5$, and $1 \mathrm{~s}$, respectively, as shown in Table 2 . The model that showed less time-consuming calculation steps (less number of calculation steps means more time consuming) with the lowest CV was chosen to further study as defined by equation (1) described in the next section.

\section{Determination of uniformity of irradiation models}

The geometries of Models A, B, C, D, E, F, G, and H were created. These geometries were meshed and divided into cell zones as described previously. The optimum irradiation time and calculated step selected from the previous section were used. In all models, the irradiation time for calculating the step of the UV-C conveyor configuration models was fixed at $1 \mathrm{~s}$. The total number of movement steps (or total irradiation time) was set at $6 \mathrm{~s}$. The movement speed of the conveyor was fixed at $0.1795 \mathrm{~m} / \mathrm{s}$. The total irradiated UV-C dose distribution was estimated by accumulating the incident UV-C dose on the surface of strawberries.

To determine the optimum model that showed the best irradiation uniformity, the coefficient of variation (CV) was employed. CV is a normalized index of dispersion of a probability distribution and it can be defined as follows:

$$
C V=\frac{\sigma}{\mu}
$$

where $\sigma$ is the standard deviation and $\mu$ is the mean intensity of surface incident radiation $\left(\mathrm{W} / \mathrm{m}^{2}\right)$.

The model that showed the best uniformity of irradiation with the lowest CV value was selected for further study.

\section{Determination of inactivation model of $P$. digi- tatum}

Microbial inactivation on the surface of strawberries

Table 1. The physical surfaces and domain walls parameters

\begin{tabular}{cccccc}
\hline Name & Types & Internal emissivity & Radiation & Diffuse fraction & Temperature \\
\hline Opening wall & Pressure-outlet & - & - & - & $10 \mathrm{~K}$ \\
Cover & Wall & 0.35 & Opaque & 0.5 & - \\
Lamp & Wall & 0.89 & Semi-transparent & 1 & $10 \mathrm{~K}$ \\
Strawberry & Wall & 0.95 & Opaque & 0.5 & - \\
Film & wall & 1 & Semi-transparent & 0 & - \\
\hline
\end{tabular}

Table 2. The calculation step parameters and $C V$

\begin{tabular}{lccc}
\hline & Model C & Model C-1 & Model C-2 \\
\hline Calculation time for each steps (s) & 1 & 0.5 & 0.1 \\
Total step & 6 & 12 & 60 \\
Total irradiation time (s) & $(1 \times 6)=6$ & $(0.5 \times 12)=6$ & $(0.1 \times 60)=6$ \\
$C V$ & 0.465 & 0.461 & 0.459 \\
\hline
\end{tabular}


treated with UV-C irradiation was evaluated in accordance with a previous study Tanaka et al. (2016). The inactivation kinetic model with a radiation transfer model was used in this study. $P$. digitatum is a common microorganism that causes postharvest fungal disease (Tanaka et al., 2016, Olmedo et al., 2017, García-Martín et al., 2018). The first kinetic model for inactivation of $P$. digitatum was applied to our study because this model has been proven to be a fitted model for predicting the survival curve during UV-C treatment (Tanaka et al., 2016, Trivittayasil et al., 2016)

$$
\log _{10} S=-\frac{k^{\prime} I t}{2.303}
$$

where $S$ is the survival rate, $I$ is the irradiation intensity of UV-C $\left(\mathrm{kW} / \mathrm{m}^{2}\right), t$ is the treatment time $(\mathrm{s})$, and $k$ is the modified inactivation rate constant $\left(11.98 \mathrm{~m}^{2} / \mathrm{kJ}\right)$ (Trivittayasil et al., 2015)

\section{RESULTS AND DISCUSSION}

\section{Effect of the calculated step on the incident radi- ation dose of $U V-C$ treatment}

The contours of the incident radiation dose during UV-C treatment of Model C, Model C-1, and Model C-2 are shown in Fig. 3A. The red area represents high exposure to UV-C irradiation, whereas the blue area rep-

A

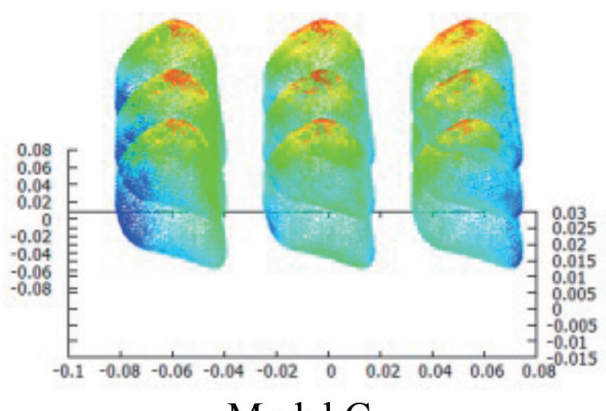

Model C
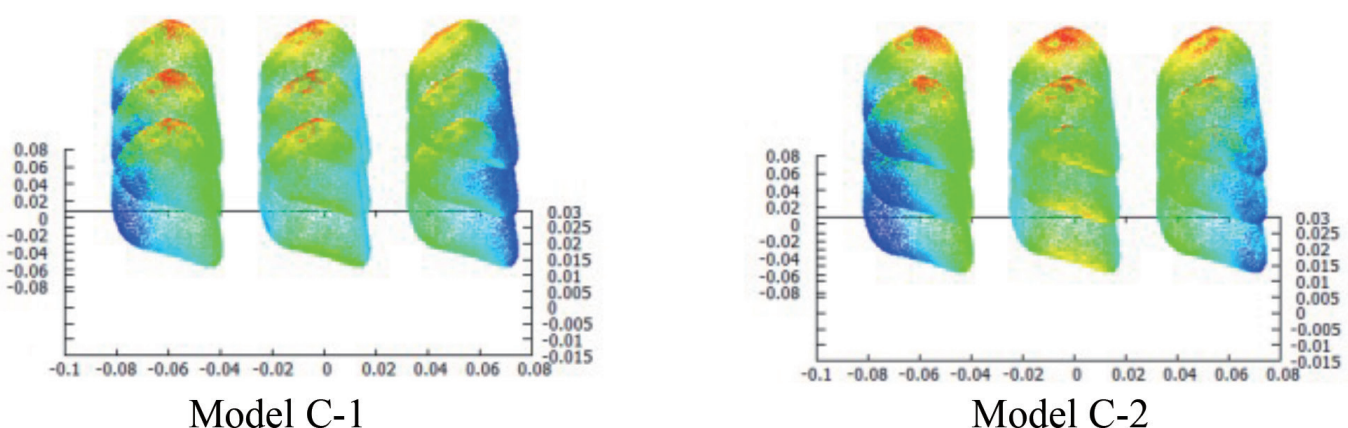

B
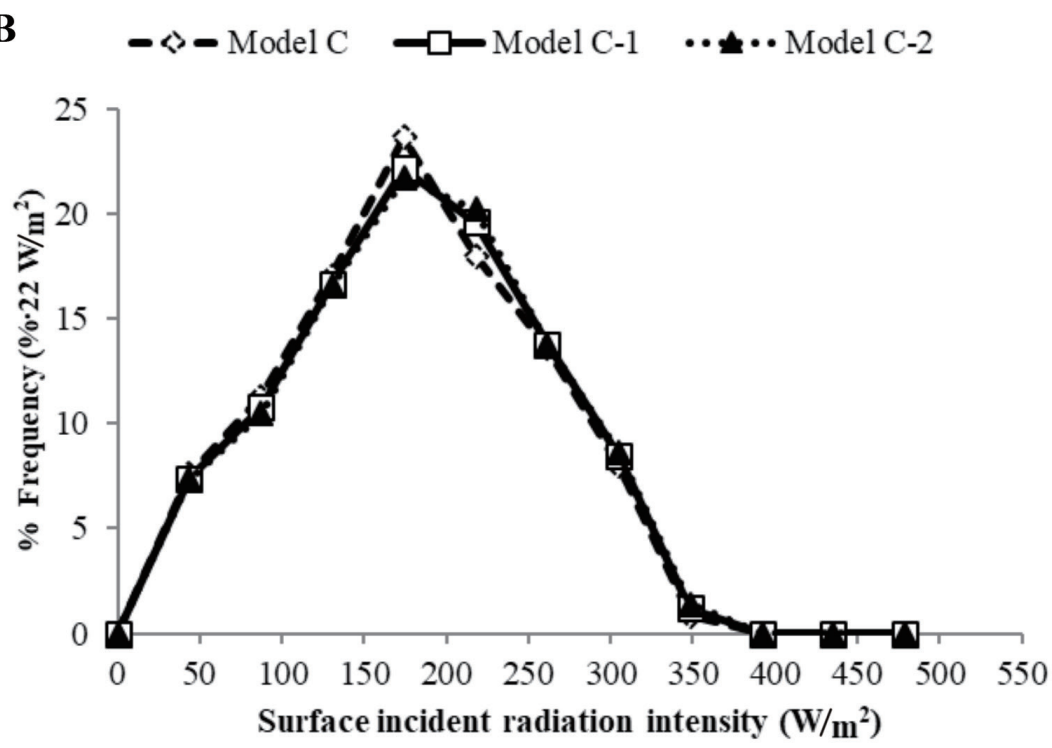

Fig. 3. The contours of the incident radiation dose during UV-C treatment (A) and the incident radiation dose during UV-C treatment (B). 


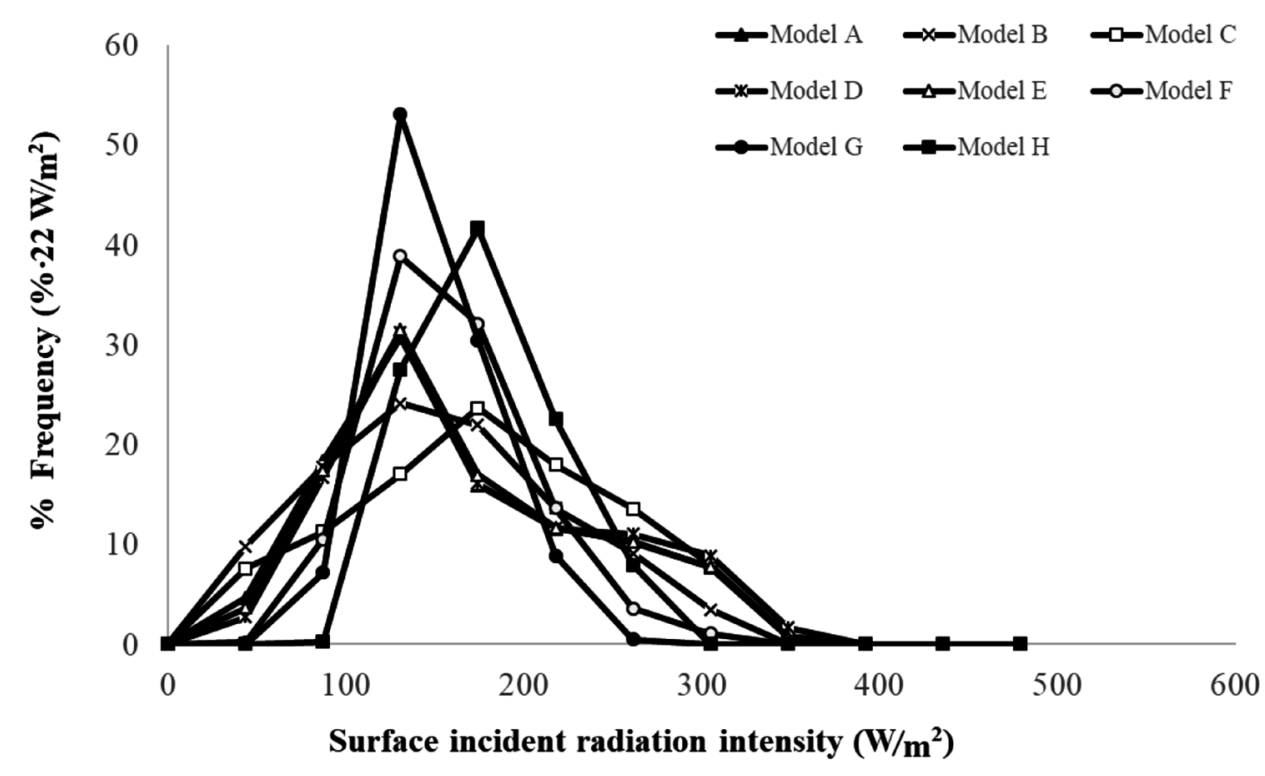

Fig. 4. The incident radiation dose during UV-C treatment of all models.

resents low exposure to UV-C irradiation. The result was that the overexposed area was mostly found on the top sides of the strawberries exposed to UV-C irradiation. The irradiation dose frequency distribution of all samples is shown in Fig. 3B. The results indicated that no difference was observed in irradiation dose in all models. The highest CV was observed in Model C-2 followed by Model C-1 and Model C (Table 2). Because the size of the calculation step was finer, the CV value tended to decrease, resulting in these models showing greater accuracy and closer to the real situation. However, even though small calculation steps provided more accurate solutions than larger calculation steps, the small size calculation steps required a long time to process. Thus, because the $\mathrm{CV}$ value of Model $\mathrm{C}$ was intimately related to Models $\mathrm{C}-1$ and $\mathrm{C}-2$, it was selected for further study. This meant that we could obtain the same calculation result for predicting the incident radiation dose of UV-C at the surface of strawberries irrespective of which model was used.
Evaluation of the simulation effect of $U V-C$ conveyor models on the uniform irradiation distribution at 6 s treatment time

Understanding of the UV light intensity distribution in a treatment chamber is of great importance in terms of the application of the right conditions that can maximize the inactivation efficiency (Hakguder Taze and Unluturk, 2018). The uniform irradiation distribution results of the simulation are presented in Fig. 4. The different histograms of each model can be categorized visually. A narrow distribution range is desired because it contributes to reducing the risk of overexposure and underexposure. Table 3 shows the maximum, minimum, mean, and CV of the accumulated incident UV-C dose distributions of all models. The results of the evaluation were as follows:

The patterns of lamps and their covers played an important role in the uniformity of UV-C distribution. The mean UV-C intensities of all models were found in range of $64.25-79.27 \mathrm{~J} / \mathrm{m}^{2}$. The highest mean UV-C

Table 3. UV-C intensity and \% inactivation of Penicillium digitatum of model A, B and $\mathrm{C}$ at 6 s treatment

\begin{tabular}{|c|c|c|c|c|c|c|c|}
\hline \multirow{2}{*}{ Model } & \multicolumn{3}{|c|}{ UV-C intensity $\left(\mathrm{kJ} / \mathrm{m}^{2}\right)$} & \multirow{2}{*}{$C V$} & \multicolumn{3}{|c|}{$\%$ Inactivation } \\
\hline & Maximum & Minimum & Mean & & Maximum & Minimum & Mean \\
\hline Model A & 162.56 & 4.77 & 71.99 & 0.50 & 85.87 & 5.55 & 57.72 \\
\hline Model B & 150.61 & 4.70 & 66.48 & 0.51 & 83.55 & 5.47 & 54.86 \\
\hline Model C & 163.45 & 3.30 & 79.26 & 0.47 & 85.90 & 3.88 & 61.32 \\
\hline Model D & 165.79 & 6.31 & 75.70 & 0.48 & 86.28 & 7.29 & 59.63 \\
\hline Model E & 162.66 & 6.45 & 72.67 & 0.49 & 85.76 & 7.44 & 58.14 \\
\hline Model F & 153.45 & 19.66 & 68.57 & 0.32 & 84.10 & 20.99 & 56.03 \\
\hline Model G & 116.11 & 26.73 & 64.25 & 0.26 & 75.13 & 27.40 & 53.69 \\
\hline Model H & 131.79 & 37.14 & 79.27 & 0.24 & 79.39 & 35.92 & 61.32 \\
\hline
\end{tabular}


intensity with the lowest $C V$ was observed in Model $\mathrm{H}$. It was postulated that because the configuration of Model $\mathrm{H}$ comprising lamps and covers that were placed at the corners of the chamber with reflectors placed between these lamps, resulting in this model providing a better UV-C distribution than other models. The lowest UV-C intensity was observed in Model G, which had almost the same configuration as Model $\mathrm{H}$, but Model $\mathrm{G}$ had no reflectors. It was noted that the presence or absence reflectors in the chamber had a direct effect on UV-C distribution. The results were confirmed in Model A and Model D, in which the reflectors present in Model D provided better UV-C distribution than in Model A. Moreover, the position of the reflectors in the chamber showed a significant impact on UV-C uniformity, which was observed in Models D and E. Reflectors located at the top of chamber produced more pronounced uniformity than those located at the bottom of the chamber. The gap between the bottom lamps and sample tended to affect the uniformity. The small gap in Model C provided the more uniformity than in Models $\mathrm{A}$ and $\mathrm{B}$. Thus, several factors were involved in UV-C distribution when each model was created.

\section{Evaluation uniformity of irradiation and \% inac- tivation of $P$. digitatum in the simulation system}

In this analysis, we studied the effect of treatment times ( $6 \mathrm{~s}, 1 \mathrm{~min}$, and $6 \mathrm{~min}$ ) of all Models to inactivate $P$. digitatum in the simulation system. Generally, a three- log reduction (99.9\%) is recognized as the target for mold inactivation in the treatment of fruits (Trivittayasil et al., 2016). With a $6 \mathrm{~s}$ treatments with UV-C (Table 3), all models showed a \% inactivation mean of 53.69$61.32 \%$. This evidence indicated insufficient treatment times, because the \% inactivation of $P$. digitatum did not reach a three-log reduction (99.9\%). Thus, it was expected that an increase in the treatment time as needed in order to achieve the three log reduction in \% inactivation rate for all models. After increasing the treatment time from $6 \mathrm{~s}$ to $1 \mathrm{~min}$ (Table 4), the average $\%$ inactivation showed satisfactory values (99.9\%), which coincided with the increasing UV-C intensity in all models. The results were in accordance with Hakguder Taze and Unluturk (2018), who reported that UV dose values changed from 0 to $48.45 \mathrm{~kJ} / \mathrm{m}^{2}$ when treatment times increased from 0 and $25 \mathrm{~min}$. The highest value of the minimum \% inactivation was found in Model $\mathrm{H}$. It might be postulated that depending on their configurations, specific models could provide higher intensity distributions than other models. Normally, the adequate radiation dose in order to maintain quality and ensure microorganism safety for strawberry is between 0.5 to $4.0 \mathrm{~kJ} / \mathrm{m}^{2}$ (Tanaka et al., 2016). Liu et al. (2018) found that the total phenolics, phenolic acids, and flavonoids contents were more pronounced when mature-green tomato fruit were irradiated with UV-C at $4 \mathrm{~kJ} / \mathrm{m}^{2}$ and stored in the dark at $13^{\circ} \mathrm{C}$ and $95 \%$ RH. Ortiz Araque et al. (2018) revealed that strawberry pre-storage UV-C

Table 4. UV-C intensity and \% inactivation of Penicillium digitatum of model A-H at 1 min treatment time

\begin{tabular}{|c|c|c|c|c|c|c|c|}
\hline \multirow{2}{*}{ Model } & \multicolumn{3}{|c|}{$\mathrm{UV}-\mathrm{C}$ intensity $\left(\mathrm{kJ} / \mathrm{m}^{2}\right)$} & \multirow{2}{*}{$C V$} & \multicolumn{3}{|c|}{$\%$ Inactivation } \\
\hline & Maximum & Minimum & Mean & & Maximum & Minimum & Mean \\
\hline Model A & 1.63 & 0.05 & 0.72 & 0.05 & 100.00 & 43.52 & 99.98 \\
\hline Model B & 1.51 & 0.05 & 0.66 & 0.05 & 100.00 & 43.03 & 99.96 \\
\hline Model C & 1.63 & 0.03 & 0.79 & 0.04 & 100.00 & 32.66 & 99.99 \\
\hline Model D & 1.66 & 0.06 & 0.76 & 0.04 & 100.00 & 53.08 & 99.99 \\
\hline Model E & 1.63 & 0.06 & 0.73 & 0.04 & 100.00 & 53.86 & 99.98 \\
\hline Model F & 1.53 & 0.20 & 0.69 & 0.03 & 100.00 & 90.52 & 99.97 \\
\hline Model G & 1.16 & 0.27 & 0.64 & 0.02 & 100.00 & 95.93 & 99.95 \\
\hline Model H & 1.32 & 0.37 & 0.79 & 0.02 & 100.00 & 98.83 & 99.99 \\
\hline
\end{tabular}

Table 5. UV-C intensity and \% inactivation of Penicillium digitatum of model A-H at 6 min treatment time

\begin{tabular}{|c|c|c|c|c|c|c|c|}
\hline \multirow{2}{*}{ Model } & \multicolumn{3}{|c|}{ UV-C intensity $\left(\mathrm{kJ} / \mathrm{m}^{2}\right)$} & \multirow{2}{*}{$C V$} & \multicolumn{3}{|c|}{$\%$ Inactivation } \\
\hline & Maximum & Minimum & Mean & & Maximum & Minimum & Mean \\
\hline Model A & 9.75 & 0.29 & 4.31 & 0.01 & 100.00 & 96.75 & 100.00 \\
\hline Model B & 9.04 & 0.28 & 3.98 & 0.01 & 100.00 & 96.58 & 100.00 \\
\hline Model C & 9.81 & 0.20 & 4.76 & 0.01 & 100.00 & 90.67 & 100.00 \\
\hline Model D & 9.95 & 0.38 & 4.54 & 0.01 & 100.00 & 98.93 & 100.00 \\
\hline Model E & 9.76 & 0.39 & 4.36 & 0.01 & 100.00 & 100.00 & 100.00 \\
\hline Model F & 9.21 & 1.18 & 4.11 & 0.01 & 100.00 & 100.00 & 100.00 \\
\hline Model G & 6.97 & 1.60 & 3.85 & 0.00 & 100.00 & 100.00 & 100.00 \\
\hline Model H & 7.91 & 2.23 & 4.76 & 0.00 & 100.00 & 100.00 & 100.00 \\
\hline
\end{tabular}


treatments at doses ranging from 0.2 to $4.2 \mathrm{~kJ} / \mathrm{m}^{2}$ reduced decay. It was noteworthy that the average UV-C intensity with a 1 min treatment time in all models was $0.64-0.79 \mathrm{~kJ} / \mathrm{m}^{2}$, which was in the recommended range. However, all of models with $1 \mathrm{~min}$ treatment time provided an unsatisfactory minimum \% inactivation value ( $<99.9 \%$ reduction). Thus, a 6 min treatment time was employed to improve the effectiveness of UV-C treatment (Table 5). Focusing on higher treatment time (6 min), 100\% inactivation was shown in all models, in which the average UV intensity in all models (3.85$4.76 \mathrm{~kJ} / \mathrm{m}^{2}$ ) was in the recommended range. Nevertheless, even increasing to a higher treatment time, different UV-C intensities still appeared because of the different configurations in the models. Tanaka et al. (2016) reported that, the model with four lamps installed in parallel to the direction of movement provided uniform dose distribution. This result suggested that the enhanced UV-C treatment time had an effect to inactivate $P$. digitatum. Additionally, Model H should be recommended for use in further studies to obtain higher efficiency in $P$. digitatum inhibition.

\section{CONCLUSION}

UV-C radiation intensity distributions on the surface of strawberries were predicted using a variety of configurations, and the survival ratio of $P$. digitatum was estimated based on the irradiation intensity distribution governed by the UV-C model. The addition of reflectors to the model provided better uniformity. The best configuration model providing the most uniform irradiation intensity distribution with the lowest CV included lamps and theirs cover located in the corners of the chamber and reflectors placed between the lamps. Adjustment of the calculation step to be finer had a negligible effect on the simulation processing time. Based on treatment time, increasing treatment to 1 and 6 min resulted in increased \% inactivation of $P$. digitatum, which reached a $3 \log$ reduction. Thus, the use of CFD techniques was an effective for modeling and simulating UV-C dose distribution.

Acknowledgments: The work was supported by the JICA Innovative Asia scholarship [Project no. D1706943]

\section{REFFERENCES}

Ambaw, A., Verboven, P., Defraeye, T., Tijskens, E., Schenk, A., Opara, U. L. and Nicolai, B. M. 2013 Porous medium modeling and parameter sensitivity analysis of $1-\mathrm{MCP}$ distribution in boxes with apple fruit. J. Food Eng., 119: 13-21

Barbosa-Cánovas, G. V., Ghani Albaali, A., Juliano, P. and Knoerzer, K. 2011 Introduction to innovative food processing technologies: background, advantages, issues, and need for multiphysics modeling. In Knoerzer, K., Juliano, P., Roupas, P. and Versteeg, C. (eds.) Innovative food processing technologies: Advances in Multiphysics simulation. John Wiley \& Sons, Ltd. and Institute of Food Technologists

Cârlescu, P.-M., Arsenoaia, V., Roşca, R. and Ţenu, I. 2017 CFD simulation of heat and mass transfer during apricots drying. LWT-Food Sci. Technol., 85: 479-486

García-Martín, J. F., Olmo, M. and García, J. M. 2018 Effect of ozone treatment on postharvest disease and quality of different citrus varieties at laboratory and at industrial facility. Posthavest Biol. Tec., 137: 77-85

Gutiérrez, D. R., Chaves, A. R. and Rodríguez, S. D. C. 2018 UV-C and ozone treatment influences on the antioxidant capacity and antioxidant system of minimally processed rocket (Eruca sativa Mill.). Posthavest Biol. Tec., 138: 107-113

Hakguder Taze, B. and Unluturk, S. 2018 Effect of postharvest UV-C treatment on the microbial quality of 'Şalak' apricot. Sci. Hortic., 233: 370-377

Hou, B., Ye, R., Huang, Y., Wang, X. and Zhang, T. 2018 A CFD model for predicting the heat transfer in the industrial scale packed bed. Chinese J. Chem. Eng., 26: 228-237

Knoerzer, K., Juliano, P., Roupas, P. and Versteeg, C. 2011. Innovative food processing technologies: advances in multiphysics simulation, John Wiley \& Sons

Kumarasamy, K., An, J., Yang, J. and Yang, E.-H. 2016 Numerical techniques to model conduction dominant phase change systems: A CFD approach and validation with DSC curve. Energy Build., 118: 240-248

Liu, C., Zheng, H., Sheng, K., Liu, W. and Zheng, L. 2018 Effects of postharvest UV-C irradiation on phenolic acids, flavonoids, and key phenylpropanoid pathway genes in tomato fruit. Sci. Hortic., 241: 107-114

Lu, J., Charles, M. T., Vigneault, C., Goyette, B. and Raghavan, G. S. V. 2010 Effect of heat treatment uniformity on tomato ripening and chilling injury. Posthavest Biol. Tec., 56: 155-162

Olmedo, G. M., Cerioni, L., Gonzalez, M. M., Cabrerizo, F. M., Rapisarda, V. A. and Volentini, S. I. 2017 Antifungal activity of beta-carbolines on Penicillium digitatum and Botrytis cinerea. Food Microbiol., 62: 9-14

Ortiz Araque, L. C., Rodoni, L. M., Darré, M., Ortiz, C. M., Civello, P. M. and Vicente, A. R. 2018 Cyclic low dose UV-C treatments retain strawberry fruit quality more effectively than conventional pre-storage single high fluence applications. LWT-Food Sci. Technol., 92: 304-311

Pan, Y., Sun, D.-W., Cheng, J.-H. and Han, Z. 2018 Non-destructive detection and screening of non-uniformity in microwave sterilization using hyperspectral imaging analysis. Food Anal. Methods, 11: 1568-1580

Park, H. and Yoon, W. 2018 Computational Fluid Dynamics (CFD) modelling and application for sterilization of foods: A Review. Processes, 6: 62

Pu, Y.-Y. and Sun, D.-W. 2017 Combined hot-air and microwave-vacuum drying for improving drying uniformity of mango slices based on hyperspectral imaging visualisation of moisture content distribution. Biosyst. Eng., 156: 108-119

Tanaka, F., Nashiro, K., Trivittayasil, V. and Uchino, T. 2016 Simulation of UV-C dose distribution and inactivation of mold spore on strawberries in a conveyor system. J. Food Sci. Technol., 22: 461-466

Trivittayasil, V., Nashiro, K., Tanaka, F., Hamanaka, D. and Uchino, T. 2015 Inactivation characteristics and modeling of mold spores by UV-C radiation based on irradiation dose. J. Food Sci. Technol., 21: 365-370

Trivittayasil, V., Tanaka, F. and Uchino, T. 2016 Simulation of UV-C intensity distribution and inactivation of mold spores on strawberries. J. Food Sci. Technol., 22: 185-192

Zhao, C.-J., Han, J.-W., Yang, X.-T., Qian, J.-P. and Fan, B.-L. 2016 A review of computational fluid dynamics for forced-air cooling process. Appl. Energ., 168: 314-331 